\title{
Effect of temperature on the coherence time of a parabolic quantum dot qubit
}

\author{
Wei Xiao* and Hong-Wei Wang \\ Department of Basic Sciences, University of Informational Science and Technology of Beijing \\ Beijing 100101, China \\ E-mail: xiaowei1123@126.com
}

Received June 10, 2014, revised September 11, 2014,

published online January 27, 2015

\begin{abstract}
The effects of the temperature on the coherence time of a parabolic quantum dot (PQD) qubit are investigated by using the variational method of Pekar type. We obtain the ground and the first excited states' eigenenergies and the corresponding eigenfunctions of an electron strongly coupled to bulk longitudinal optical phonons in the PQD. This two-level PQD system may be employed as a single qubit. The phonon spontaneous emission causes the decoherence of the qubit. We find that the coherence time will decrease with increasing temperature. It is an increasing function of the effective confinement length, whereas it is decreasing one of the polaron radius. We find that by changing the temperature, the effective confinement length and the polaron radius one can adjust the coherence time. Our research results would be useful for the design and implementation of the solid-state quantum computation.
\end{abstract}

PACS: 71.38.-k Polarons and electron-phonon interactions;

73.21.La Quantum dots;

63.20.kd Phonon-electron interactions.

Keywords: parabolic quantum dot, qubit, coherence time.

\section{Introduction}

In recent years, investigations of the quantum information (QI) and quantum computation (QC) in quantum dot (QD) systems have attracted considerable interest of not only experimenters but also theorists. This interest is based on prospects for the application of realizing quantum computer and QI processing. At present, there are many experiment methods [1-3] and theoretical studies [4-6] on QI and QC in the QD systems. It is clear that QC with a large significant number of qubits would be more realizable in the QD systems, in which two-level (TL) system may be used as a single qubit. However, this quantum system is very frail due to the interactions between the quantum memories and their surroundings. Therefore, quantum coherence plays a crucial role in the QC. The coherence of qubit is crucial to the investigations of QC, where the coherence time is one of the physical quantities representing the properties of coherence. This problem was solved by different authors by prolonging the quantum coherence time in experiments. For example, based on the spin modelocking and spin echo techniques, Varwig et al. [7] studied the temperature dependence of the coherence time of hole spins confined in self-assembled (In,Ga)As/GaAs QDs. Using ultrafast time-resolved Faraday ellipticity at room temperature, Zhang et al. [8] investigated the electron spin coherences in colloidal CdSe QDs with zinc blende structure. Borri et al. [9] used a highly sensitive four-wave mixing to measure the dephasing time which is about several hundred picoseconds at low temperature in the ground state transition of strongly confined InGaAs QDs. In theory, Bonadeo et al. [10] investigated coherent optical control of the quantum state of a single QD by using the excitonic wave function. Fischer et al. [11] discussed spin interactions, relaxation and decoherence in QDs. The properties of qubit with temperature, have been studied. Chen et al. [12] used the variational method of Pekar type (VMPT) to investigate the effect of temperature on the PQD qubit in the electric field. Sun et al. [13] studied the effects of temperature and magnetic field on a quantum rod qubit. Wang

\footnotetext{
* Corresponding author. 
et al. [14] obtained the decoherence time of the PQD qubit by using VMPT. Based on a VMPT, Sun et al. [15] calculated the effect of magnetic field on the coherence time of a PQD qubit. To obtain more information about the effects of temperature, the qubit and its coherence, the reader can refer to [16-18]. Such solutions would be useful to study the decoherence of the QD qubit. However, the temperature dependence of the coherence time of qubit in a PQD hasn't been reported. In the present paper, based on the VMPT, we study the effects of the temperature, the effective confinement length and the polaron radius on the coherence time in the PQD qubit.

\section{Theoretical model and calculations}

We consider the system in which the electrons are bounded by the parabolic potential. The electrons are confined much stronger in one direction (taken as the $z$ direction) than in the other two directions. Therefore, we will focus on the condition of the electron and longitudinal optical (LO) phonon moving only on the $X Y$ plane. We assume that the confining potential in a single QD is parabolic:

$$
V(\rho)=\frac{1}{2} m \omega_{0}^{2} \rho^{2},
$$

where $m$ is the band mass of electron, $\boldsymbol{\rho}=(x, y)$ is the coordinate vector of a two-dimensional and $\omega_{0}$ is the confinement strength of QD. The Hamiltonian of an electronphonon interaction system can be written as

$$
\begin{aligned}
H=- & \frac{\hbar^{2} \nabla_{\rho}^{2}}{2 m}+\sum_{\mathbf{q}} \hbar \omega_{L O} a_{\mathbf{q}}^{+} a_{\mathbf{q}}+\frac{1}{2} m \omega_{0}^{2} \rho^{2}+ \\
& +\sum_{\mathbf{q}}\left[V_{q} a_{\mathbf{q}} \exp (\text { iq } \cdot \mathbf{r})+\text { h.c. }\right],
\end{aligned}
$$

where $a_{\mathbf{q}}^{+}\left(a_{\mathbf{q}}\right)$ denotes the creation (annihilation) operator of the bulk LO phonons with wave vector $\mathbf{q} ; \mathbf{r}=(\rho, z)$ is the position vector of the electron; $V_{q}$ and $\alpha$ are

$$
\begin{aligned}
& V_{q}=i\left(\frac{\hbar \omega_{L O}}{q}\right)\left(\frac{\hbar}{2 m \omega_{L O}}\right)^{\frac{1}{4}}\left(\frac{4 \pi \alpha}{v}\right)^{\frac{1}{2}}, \\
& \alpha=\left(\frac{e^{2}}{2 \hbar \omega_{L O}}\right)\left(\frac{2 m \omega_{L O}}{\hbar}\right)^{\frac{1}{2}}\left(\frac{1}{\varepsilon_{\infty}}-\frac{1}{\varepsilon_{0}}\right),
\end{aligned}
$$

where $v$ is the volume of the crystal, $\varepsilon_{0}$ and $\varepsilon_{\infty}$ are, respectively, the static and high-frequency dielectric constant of the crystal. Following the VMPT [19,20], the trial wave function of the electron-phonon system can be separated into two parts, which separately describes the electron and phonon. Then the system's trial the ground and the first excited states' (GFES') wave functions may be chosen [13,15]

$$
\left|\varphi_{0}\right\rangle=|0\rangle|\xi(z)\rangle\left|0_{\mathrm{ph}}\right\rangle=\frac{\lambda_{0}}{\sqrt{\pi}} \exp \left[-\frac{\lambda_{0}^{2} \rho^{2}}{2}\right]|\xi(z)\rangle\left|0_{\mathrm{ph}}\right\rangle,
$$

$$
\begin{aligned}
\left|\varphi_{1}\right\rangle=|1\rangle|\xi(z)\rangle\left|0_{\mathrm{ph}}\right\rangle=\frac{\lambda_{1}^{2}}{\sqrt{\pi}} \rho \exp \left(-\frac{\lambda_{1}^{2} \rho^{2}}{2}\right) \times \\
\times \exp ( \pm i \phi)|\xi(z)\rangle\left|0_{\mathrm{ph}}\right\rangle,
\end{aligned}
$$

where $\lambda_{0}$ and $\lambda_{1}$ are the variational parameters, $\left|0_{\mathrm{ph}}\right\rangle$ is unperturbed zero phonon state, which satisfies $a_{\mathbf{q}}\left|0_{\mathrm{ph}}\right\rangle=0$. $|\xi(z)\rangle$ is the wave function of an electron in the $z$ direction, since the electrons are much more strongly confined in $z$ direction than in other two directions and considered to be confined in an infinite simally narrow layer, so does $\langle\xi(z) \mid \xi(z)\rangle=\delta(z) .|0\rangle$ and $|1\rangle$ are trial GFES' wave functions of the electron in the $\rho$ direction. The above equations satisfy the following normalized relations:

$$
\left\langle\varphi_{0} \mid \varphi_{0}\right\rangle=1,\left\langle\varphi_{0} \mid \varphi_{1}\right\rangle=0,\left\langle\varphi_{1} \mid \varphi_{0}\right\rangle=0,\left\langle\varphi_{1} \mid \varphi_{1}\right\rangle=1 \text {. }
$$

By minimizing the expectation value of the Hamiltonian, we then obtain the electron ground-state energy $E_{0}=\left\langle\varphi_{0}|H| \varphi_{0}\right\rangle$ and the first excited state energy $E_{1}=\left\langle\varphi_{1}|H| \varphi_{1}\right\rangle$. The GFES' energies of electron in a PQD can be written as

$$
E_{0}\left(\lambda_{0}\right)=\frac{\hbar^{2} \lambda_{0}^{2}}{2 m}+\frac{\hbar^{2}}{2 \lambda_{0}^{2} m l_{0}^{4}}+\frac{m \omega_{c}^{2}}{8 \lambda_{0}^{2}}-\frac{1}{2}(2 \pi)^{1 / 2} \alpha \hbar \omega_{L O} r_{0} \lambda_{0},
$$

and

$$
E_{1}\left(\lambda_{1}\right)=\frac{\hbar^{2} \lambda_{1}^{2}}{2 m}+\frac{\hbar^{2}}{m \lambda_{1}^{2} l_{0}^{4}}+\frac{m \omega_{c}^{2}}{4 \lambda_{1}^{2}}-\frac{11}{32} \alpha \hbar \omega_{L O}(2 \pi)^{1 / 2} r_{0} \lambda_{1},
$$

where $l_{0}=\left(\hbar / m \omega_{0}\right)^{1 / 2}$ is the effective confinement length of the PQD, and $r_{0}=\left(\hbar / 2 m \omega_{L O}\right)^{1 / 2}$ is the polaron radius. We can obtain $\lambda_{0}$ and $\lambda_{1}$ by using the variational method and thus to get the eigen levels and eigen wave functions. So, a TL system as a single qubit is built up. The superposition state of electron can be expressed as

$$
\left|\psi_{01}\right\rangle=\frac{1}{\sqrt{2}}(|0\rangle+|1\rangle)
$$

Under the dipole approximation, based on the Fermi golden rule [21], the spontaneous emission rate can be written in the following form:

$$
\begin{gathered}
\tau^{-1}=\frac{e^{2} \Delta E}{3 \pi \varepsilon_{0} \hbar^{2} m^{2} C^{3}} \sqrt{\frac{\varepsilon}{\varepsilon_{0}}}|\langle 0|r| 1\rangle|^{2}= \\
=\frac{e^{2} \Delta E}{3 \pi \varepsilon_{0} \hbar^{2} m^{2} C^{3}} \sqrt{\frac{\varepsilon}{\varepsilon_{0}}} \frac{16 \lambda_{0}^{2} \lambda_{1}^{4}}{\left(\lambda_{0}^{2}+\lambda_{1}^{2}\right)^{4}}
\end{gathered}
$$

where $C$ is the speed of light in vacuum, $\varepsilon\left(\varepsilon_{0}\right)$ is the material (vacuum) dielectric constant, $\Delta E=E_{1}-E_{0}$ is the energy separation between the GFES, $\tau$ is the coherence time. 
The optical phonons mean number in superposition state around the electron is

$$
N=\frac{1}{4}(2 \pi)^{1 / 2} \alpha r_{0} \lambda_{0}+\frac{11}{64}(2 \pi)^{1 / 2} \alpha r_{0} \lambda_{1} .
$$

\section{Temperature effect}

At finite temperature, the electron-phonon system is no longer entirely in the ground state. The lattice vibrations excite not only real phonon but also the electron confined in the parabolic potential. The properties of the polaron are statistical averages of various states. According to the quantum statistics theory, the statistical average number of the bulk LO phonons is given by

$$
\bar{N}=\left[\exp \left(\frac{\hbar \omega_{L O}}{k_{B} T}\right)-1\right]^{-1},
$$

where $k_{B}$ is the Boltzmann constant and $T$ denotes the temperature of the system. With the consideration mentioned above, the values of $\lambda_{0}$ and $\lambda_{1}$ determined by Eq. (11) relates not only to the value of $N$ but also to the value of $\bar{N}$, which means that Eqs. (11) and (12) should be self-consistently calculated to obtain the relationship of $\lambda_{0}$ and $\lambda_{1}$ with the temperature $T$. From Eqs. (7), (8), (10), and (11) we can see that the eigen levels, the coherence time and the optical phonons mean number of the electron in a PQD are all dependent on the variational parameters $\lambda_{0}$ and $\lambda_{1}$, so they are connected with the temperature.

\section{Numerical results and discussion}

Numerical calculations are carried out on a RbCl PQD. The experiment parameters used in the calculation are $\hbar \omega_{L O}=21.45 \mathrm{meV}, m=0.432 m_{0}, \alpha=3.81$ [22]. The effects of the temperature, the effective confinement length and the polaron radius on the coherence time, which are extracted from a numerical evaluation, are shown in Figs. 1 and 2.

Figure 1 describes the relationship between the coherence time $\tau$ of the TL quantum system versus the temperature $T$ and the effective confinement length $l_{0}$ for $\mathrm{RbCl}$ crystal with the polaron radius $r_{0}=4.0 \mathrm{~nm}$. The coherence time $\tau$ as a function of the temperature and the polaron radius $r_{0}$ is depicted in Fig. 2 for $\mathrm{RbCl}$ crystal with the effective confinement length $l_{0}=2.0 \mathrm{~nm}$. From the two figures we can see that the coherence time decrease with the increase of the temperature. This is because that the influence of the temperature in the PQD is weaker in the first excited state than that in the ground state and the increase in the first excited state is smaller than that in the ground state with increasing temperature. So the increasing of the energy spacing between the first excited and the ground states causes the reduction of the coherence time. This result is in agreement with the results of Makhlin

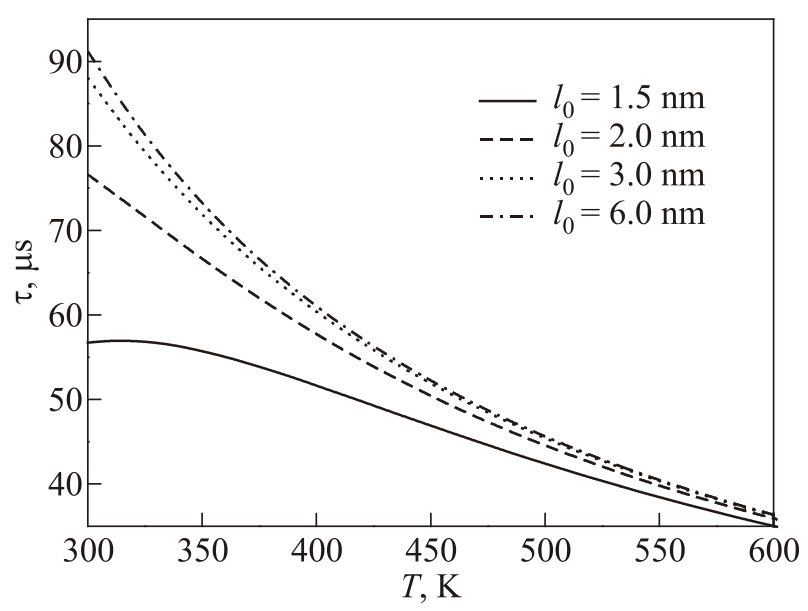

Fig. 1. The coherence time $\tau$ as a function of the temperature $T$ and effective confinement length $l_{0}$ for RbCl crystal.

et al. [23] and Wang et al. [24]. We also find that the coherence time is an increasing function of the effective confinement length, whereas it is a decreasing one of the polaron radius. The reason is that the electron energy and the interaction energy of the electron-phonon, which takes phonon as a medium, are enhanced when the particles motion range becomes smaller with the decrease of the effective confinement length. The energy spacing between the GFES increases with decreasing effective confinement length and the coherence time decreases. These phenomena can be attributed to the interesting quantum size confining effect. The reason of the coherence time decrease with increasing polaron radius is that the influence of the polaron radius in the PQD is weaker in the first excited state than that in the ground state with increasing polaron radius. For this reason, the energy space between the GFES increase with increasing polaron radius and the coherence time decreases. The magnitude of the qubit's coherence time obtained in our outcome is $0-100 \mu$ s, which is in agreement with 0-100 $\mu$ s in Ref. 25 and the result of Li et al. [26],

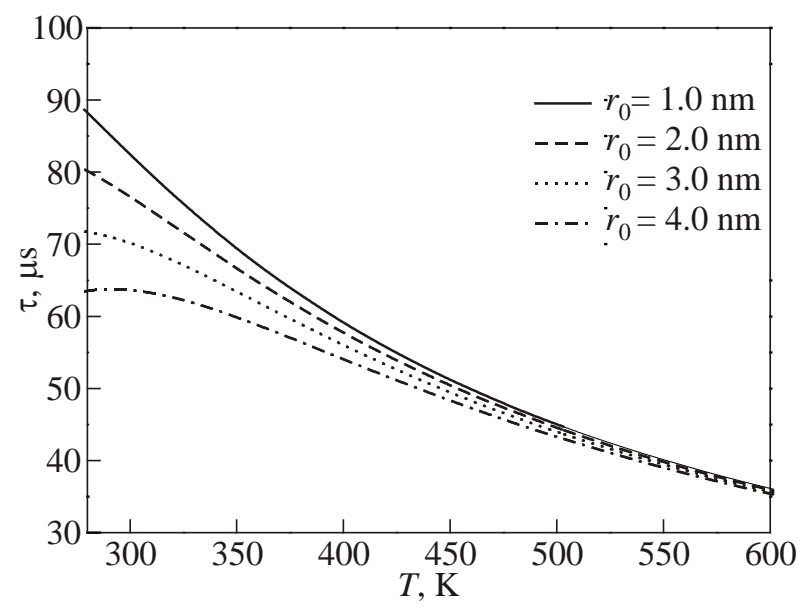

Fig. 2. The coherence time $\tau$ as a function of the temperature $T$ and polaron radius $r_{0}$ for $\mathrm{RbCl}$ crystal. 
which were obtained, respectively, by experiments and the effective mass envelope function theory.

Here we find that we can adjust the coherence time of the TL quantum system by changing the temperature, the effective confinement length and the polaron radius. Any system will be objectively suffered by the influences of temperature, etc., which is natural existence. Besides, the properties of electron in the QD system will be regulated through the way of external field. Therefore, the researches of temperature effects on the system are necessary and important to the theory and application of quantum information, which is the motivation of the present paper.

\section{Conclusions}

In this study, based on the VMPT, we investigate the effects of the temperature on the coherence time of a PQD qubit. We then obtain the GFES' eigenenergies and the corresponding eigenfunctions of an electron strongly coupled to bulk LO phonons in a PQD. This PQD may be employed as a TL qubit. The phonon spontaneous emission causes the decoherence of the qubit. It is found that the coherence time will decrease with increasing temperature. The coherence time is an increasing function of the effective confinement length, whereas it is decreasing one of the polaron radius. We find that by changing the temperature, the effective confinement length and the polaron radius one can adjust the coherence time. Our calculated results may be useful for designing the solid-state QC.

\section{Acknowledgement}

This project was supported by the National Science Foundation of China under Grant No. 11464033.

1. S. Barz, E. Kashefi, A. Broadbent, J.F. Fitzsimons, A. Zeilinger, and P. Walther, Science 335, 303 (2012).

2. K.D. Greve, P.L. McMahon, D. Press, T.D. Ladd, Y. Yamamoto, D. Bisping, C. Schneider, M. Kamp, L. Worschech, S. Höfling, A. Forchel, and Y. Yamamoto, Nature Physics 7, 872 (2011).

3. M. Leijnse and K. Flensberg. Phys. Rev. Lett. 107, 210502 (2011).

4. Q.S. Tan, Y. Dong, and L.M. Kuang, Commun. Theor. Phys. 53, 71 (2010).

5. J. Liu, Q. Wang, L.M. Kuang, and H.S. Zeng, Chinese Physics B 19, 030313 (2010).
6. R. Bose, D. Sridharan, H. Kim, G.S. Solomon, and E. Waks, Phys. Rev. Lett. 108, 227402 (2012).

7. S. Varwig, A. René, A. Greilich, D.R. Yakovlev, D. Reuter, A.D. Wieck, and M. Bayer, Phys. Rev. B 87, 115307 (2013).

8. Z. Zhang, Z. Jin, H. Ma, Y. Xu, X. Lin, G. Ma, and Xiaolan Sun, Physica E 56, 85 (2014).

9. P. Borri, W. Langbein, S. Schneider, U. Woggon, R.L. Sellin, D. Ouyang, and D. Bimberg, Phys. Rev. Lett. 87, 157401 (2001).

10. N.H. Bonadeo, J. Erland, D. Gammon, D. Park, D.S. Katzer and D.G. Steel, Science 282, 1473 (1998).

11. J. Fischer, M. Trif, W.A. Coish, and D. Loss, Solid State Commun. 149, 1443 (2009).

12. Y.J. Chen and J.L. Xiao, J. Low Temp. Phys. 170, 60 (2003).

13. Y. Sun, Z.H. Ding, and J.L. Xiao, Quantum. Inf. Process. 12, 935 (2013).

14. Z.W. Wang, J.L. Xiao, and W.P. Li, Physica B 403, 522 (2008).

15. Y. Sun, Z.H. Ding, and J.L. Xiao, J. Low Temp. Phys. 177, 151 (2014).

16. Y. Sun, Z.H. Ding, and J.L. Xiao, Physica B 444, 103 (2014).

17. G. Wendin and V.S. Shumeiko, Fiz. Nizk. Temp. 33, 957 (2007) [Low Temp. Phys. 33, 724 (2007)].

18. S.N. Shevchenko, A.N. Omelyanchouk, and E. Il'ichev, Fiz. Nizk. Temp. 38, 360 (2012) [Low Temp. Phys. 38, 283 (2012)].

19. L.D. Landau and S.I. Pekar, Zh. Eksp. Teor. Fiz. 16, 341 (1946).

20. S.I. Pekar, Untersuchungen über die Elektronen-Theorie der Kristalle, Akademie Verlag, Berlin (1954).

21. L.D. Landau and E.M. Lifshitz, Quantum Mechanics: Nonrelativistic Theory, Pergamon, London (1987).

22. Polarons in Ionic Crystals and Polar Semiconductors: Antwerp Advanced Study Institute on Fröhlich Polarons and Electron-Phonon Interaction in Polar Semiconductors, J.T. Devreese (ed.), Proceeding of the 1971, North-Holland Publ. Co., Amsterdam (1972), p. 721.

23. Y. Makhlin, G. Schon, and A. Shnirman, Rev. Mod. Phys. 73, 357 (2001).

24. Z.W. Wang and S.S. Li, J. Phys.: Condens. Matter 23, 225303 (2011).

25. C. Rigetti, S. Poletto, J.M. Gambetta, B.L.T. Plourde, J.M. Chow, A.D. Corcoles, J.A. Smolin, S.T. Merkel, J.R. Rozen, G.A. Keefe, M.B. Rothwell, M.B. Ketchen, and M. Steffen, Phys. Rev. B 86, 100506 (2012).

26. S.S. Li, J.B. Xia, J.L. Liu, F.H. Yang, Z.C. Niu, and S.L. Feng, J. Appl. Phys. 90, 6151 (2001). 